\title{
Real-Time Hand Gesture Recognition for Media Players
}

\author{
$1^{\text {st }}$ Rajib Losan Rajbonshi \\ dept. of IT \\ The Assam Kaziranga University \\ Jorhat, India \\ cs16msiit021@kazirangauniversity.in \\ $4^{\text {th }}$ Bhobesh Patir \\ dept. of IT \\ The Assam Kaziranga University \\ Jorhat, India \\ cs16msiit057@kazirangauniversity.in
}

\author{
$2^{\text {nd }}$ Amlan Jyoti Saikia \\ dept. of IT \\ The Assam Kaziranga University \\ Jorhat, India \\ cs16msiit006@kazirangauniversity.in
}

\author{
$3^{\text {rd }}$ Sankar Jyoti Saikia \\ dept. of IT \\ The Assam Kaziranga University \\ Jorhat, India \\ cs16msiit016@kazirangauniversity.in
}

\begin{abstract}
In this modern age of advancement in computing which has made the use of natural user interface much needed. Human gesture recognition is a mode of non-verbal interaction medium and can provide the most intuitive and natural way to interact with computer. The main aim is to make the interaction between human and computer as natural as the interaction between humans. The objective of this paper is to recognize the dynamic hand gesture images based on shapes and relative positions or direction of hand which is extracted from input video stream recording. These hand gestures can be used to recognition system to control multimedia applications running on computer using different gesture commands.
\end{abstract}

Index Terms - computer vision, gesture recognition, human computer interaction

\section{INTRODUCTION}

In daily life hand gestures shows a powerful means of communication. Human who have difficulty in speaking and hearing use the hand gesture to communicate with others. Gestures has been considered as an interaction technique that can deliver more natural and creative way to communicate. So, to improve the communication between humans and computer the hand gesture recognition technique have been introduced. Using gestures the user will be able to run the computer without any physical interaction. Although Human-computer interaction (HCI) is a multidisciplinary field of study related to cover relatively all forms of information technology design. Assimilating gestures in $\mathrm{HCI}$ is proving to be an imperative research area.

The sign gesture recognition can be done in two ways: static gesture and dynamic gesture [1]. While communicating, the static gesture makes use of hand shapes while the dynamic gesture makes use of the movements of the hand. Hand gesture recognition is a way of understanding and then classifying the movements by the hands. But the human hands have very complex articulations with the human body and therefore a lot of errors can arise. The problem can be resolved by applying Convolutional Neural Network (CNN) on a fairly

\author{
$5^{\text {th }}$ Monoj Kumar Muchahari \\ dept. of IT \\ The Assam Kaziranga University \\ Jorhat, India \\ 0000-0002-6758-5811
}

large dataset to achieve a good and stable accuracy. Although it is observe that huge features are mandatory for classification and recognition. Conventional models for pattern recognition cannot process natural data in raw form. Therefore, a lot of efforts are needed for mining those features.

In this paper we present a system that helps computer to recognize the hand gestures of a user, fully focusing on dynamic gestures. $\mathrm{CNN}$ is used to make the computer recognise the gestures and makes it attainable to identify the relative complex gestures of multiple users with the help of the data present in the database. Data augmentation technique is also adopted to avoid over-fitting.

The Organization of paper is as follows: section II discusses Literature study, section III Methodology, section IV Results and Conclusion is explained in section V.

\section{A. Convolutional Neural Network (CNN)}

$\mathrm{CNN}$ is an aritificial neural network that is used in image recognition which takes an input image, analyze the image with the human interaction gesture to run the system with only hand gesture interactions. CNN are able to detect patterns and images. A CNN is comprised of multiple perceptron as well as hidden layer called convolutional layer and with each convolutional layer we need to specify the number of fliters the layer should have as this filters are what detect the patterns. The CNN has four convolutional layers, three max pooling layers, two fully connected layer and one softmax output layer. CNN do not like traditional algorithm for processing each pixel of input information. But for each processing small pixel areas, strengthening the continuity of picture information, the neural network can see the graphics rather than a point. At the same time, it deepends the understanding of the image by convolution neural network. CNN have a volume filter, continually gathers information about image, every time the information collected is only a small piece of pixels of the image area. Sorting out the edge information from a collection 
of image information, and edge information from the image of General information.

\section{Literature REVIEW}

In the past few years ,many researchers have published various research and review papers on Hand gesture recognition techniques using CNN algorithm. In this section, some of the works have been discussed.

Varun et al. [2] proposed the paper named Hand gesture recognition and implementation for Disables using CNN. Here the development of the model was made in such a way that it can help the author in recognition and implementation. For such development these models will be working with Convolution neural network. In this paper, the author introduced the way for operating the data provided from the user from a web camera. The layer will be created and trained with many sample data and will try with a real-time data for checking it with many random input. It consist of more than 20,000 images with more than $12 \mathrm{gb}$. Therefore, the data which the author gave as input will be processed by a neural network and will be training that data in the CNN model. And once the data is successfully trained, it can take sample data from the user and make it recognize and make the model remember and work on the model to perform an operation when the same gesture is invoked. And at last, the author have conducted that it is able to handle some hand gestures provided by any person and helps him to identify what the gesture is. So, what we have observed is that the machine was able to understand the images and was able to identify what the images are.

Tran et al. [3] proposed the paper Real-time hand gesture spotting and recognition using RGB-D camera and 3D convolutional neural network. The paper states that compared with gestures with other body parts, hand gestures are the most efficient means of meaningful expression and serve as a common language of all humans. For example waving one's hand to express "hello" is pretty flexible. In this paper, the man goal of the author was to discover a hand gesture-based interface from depth videos. The author presented a combination of fingertip detection and a 3D convolutional neural network for hand gesture recognition from a depth camera. RGB-D provides both colour image and depth image(2D image). Despite some remarkable success, some task remains challenging occurring to its complexity as well as variable glove size between user. But the author have focused on vision-based system in which no gloves or hardware are required except for a sensor. So, that the systems are subsequently cheaper and can be easily accepted by any field.

Norah Meshari Alnaim [4] proposed the paper Hand gesture recognition using deep learning neural networks. In these paper the author propose that a system can be utilised to identify human gestures to convey information for device control. In this paper the author have develop a system to work with $2 \mathrm{D}$ and $3 \mathrm{D}$ hand gesture recognation using WT(wavelet transforms),CNN and EMD(Empirical Mode Decomposition) algorithms for feature extraction. Here the author have used data glove which has multi variety of sensors that is used to detect hand and finger motions. In the execution time their author noticed that WT is less accurate than both EMD and $\mathrm{CNN}$ algorithm. But in the end result the CNN accuracy and sensitivity is much higher then WT and EMD algorithm.

Pie Xu [5] proposed the paper A Real-time hand gesture recognition and Human-Computer interaction System. Here in this paper the author develop a system that works with real-time gesture recognition and the system supports only static gestures. Using one monocular camera the author have collected 3,200 gesture images to test with convolutional neural network and they found high accuracy of CNN in real time gesture recognition.CNN makes the work easier as it extract the gesture features manually and improve the recognition accuracy. The author have used the Kalman filter to smooth the motion of the mouse cursor controlled by the hand. In the end result their system was able to understand the hand gestures movement and function accordingly to the gesture made by the hand.

Islam et al. [6] proposed a Real-Time Hand Gesture Spotting and Recognition Using RGB-D Camera and 3D CNN and a 3D CNN for hand gesture recognition from a depth camera . In this paper, the main goal of the author was to discover a hand gesture-based interface from depth videos. The author presented a combination of finger tips detection and a 3D convolutional neural network for hand gesture recognition from a depth camera .

Thwea et al. [7] proposed a Critical Review on Hand Gesture Detection and Recognition System: . In this they have discussed various hand ges-ture detection technique and their compatibilities. They have compared different methods by evaluating their results like Dynamic time wrappig, Artificial Neural Nework etc. They have taken data from different researchers to get those results. They have intro-duced some feature extraction techniques also like Hough transfrom,

Yingxin et al. [8] proposed apaper on A Robust Hand Gesture Recognition Method via CNN. In this paper they have implemented robust hand gesture recognition system by using modified CNN. The data preprocessing has also been performed using canny edge detection which is ef-fective to remove the influence of illumination variance. According to the literature reviews, some of the paper does not include preprocess but pre-process can reduce the amount of data and remove the unwanted noise and region when pre-processing used before the feature extraction. The system achieved $98.2 \%$ of accuracy on self-constructed datasets. CNN and HMM can integrate to model dynamic hand gesture for robust spatiotemporal hand gesture recognition as future work .

Plouffe et al. [9] proposed a paper on "Static and dynamic hand gesture recognition in depth data using dynamic time warping". In this paper the authors have studied dynamic time warping algorithm for both static and dynamic hand gesture recognition on depth information. The rate of accuracy was obtained in this work is $92.4 \%$ over 55 different gestures. This paper have tried to implement a novel algorithm to improve the scanning time to identify the first pixel on the hand contour locate the fingertips by using K-curvature algorithm and then 
directional search algorithm for identification of the entire hand contour. The very complex background, illumination conditions, skin color and clothing have little impact on the resulted the proposed solution. This work was encountering some problems during testing when one of the users wore a bracelet. In this paper, as future improvements, an additional module will be added to allow for a more accurate adaption of the size of a user hand instead of the double training at different depths that is currently implemented in the system. This would improve the performance at the level of recognition stage.

Molchanov et al. [10] prposed an paper on "Hand gesture recognition with $3 \mathrm{~d}$ convolutional neural networks". In this An algorithm is introduced to recognize hand gestures using a 3D $\mathrm{CNN}$ was proposed . In this system, the basis of the recognition was challenging depth and intensity of the images. They also used data augmentation tech-nique and achieved accuracy about $77.5 \%$ on VIVA challenge dataset.

Despite some remarkable success, some task remains challenging occurring to its complexity as well as variable glove size between user but the author have focused on vision-based system in which no gloves or hardware are required except for a sensor. So, that these systems are subsequently cheaper and can be easily accepted by any field.

\section{Methodology}

In this section the functioning methods, processes and techniques are discussed.

\section{A. Data Collection}

Data sets are collected from various sources. The dataset is a large collection of labeled video clips that shows hands performing pre-defined hand gesture in front of a laptop camera or webcam. The hand gesture showing action of user varies from each other so, multiple hand gesture images are collected from several persons. The video data is provides as one large TGZ archive, splits into parts of $1 \mathrm{gb}$ max. The total file size is of $22 \mathrm{gb}$. The archive contains directories numbered from 1 to 148092 . Each directory corresponds to one video and contains JPG images. The JPG images were extracted from the original videos at 12 frames per second. The screenshots of gestures is shown in Fig. 1

1) Data Processing: In data processing we load the data from the database by providing path of the location. Each hand gesture in the 20bn-jester dataset has the same length that was created from the videos collection. These gestures are comprises of 32 frames each that has been created by taking 12 frames per second from original videos. Then we perform some augmentation to increase the accuracy for the recognition like rotation, flipping and crop-ping. We have normalized the each channel of a particular gesture's videos frames to be of zero mean and unit variance. It helps the process of the gesture classifier to converse faster than before.

2) Data Training: The process of training a CNN model is comprise of the optimization of the network's parameter to minimize the cost function for our dataset. We initialized

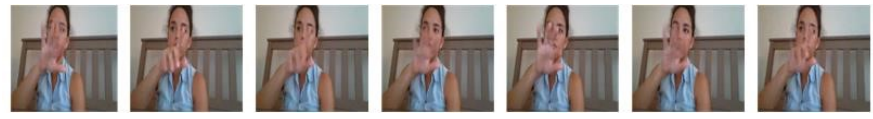

(a)

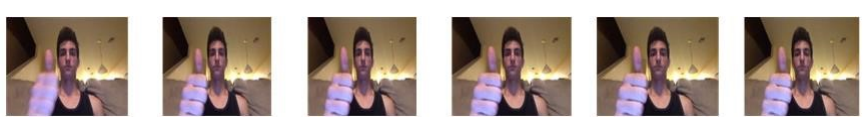

(b)

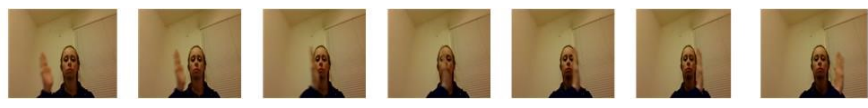

(c)

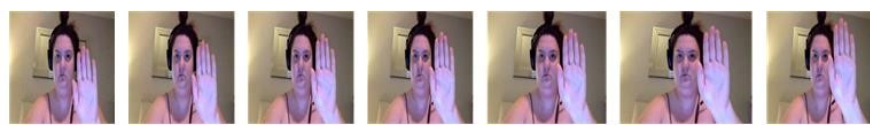

(d)

Fig. 1: Screenshots of gestures.

the weight of the convolution layers with random samples from a uniform distribution between the number of inputs and output neurons. We initiated the weight of the fully connected hidden layer with random samples with the help of normal distribution.

We trained our data with 32 frames per epoch and with 16 frames per epoch and then we com-pare their results. It is observed during the training is that the 32 frames takes much more time to train than compared to the 16 frames. But the results for both the cases are same. We also applied weight decay to all the convolution layers. After completion of each mini batches we subtracted $0.5 \%$ from the network. We observe in the process that regularization with weight decay usually resultant in better generalization for different gestures.

For the purpose of tuning the learning rate we have first initialized the rate from 0.005 and reduce it by a factor of 2 only when the cost function did not improve at least $10 \%$ for the first 40 epochs. Since the dataset is big enough for sample training we have taken only half of the data from the dataset for training. From that half data we have used 30\% data for validation.

In Fig. 2, we can observe that as the number of epoch increases means as the training is processing the accuracy is drastically increasing and it gets stable from $95 \%$ to $97 \%$. For validation also we can see the results are as good as during training. We also like to point out that these results are of training 16 frames from the dataset.

In Fig. 3, we can it has been seen that the loss of data during the training is gradually decreasing the more we train our data. It can be seen that the model is learning to take all the data that has been provided through dataset to produce the best result possible. For validation also it is shown that the loss of data is almost stands at $0 \%$ as we train our data.

3) Data Classification: In classification trained model is loaded into the system and while opening the camera to take videos. From the videos frames are collected and are appended 


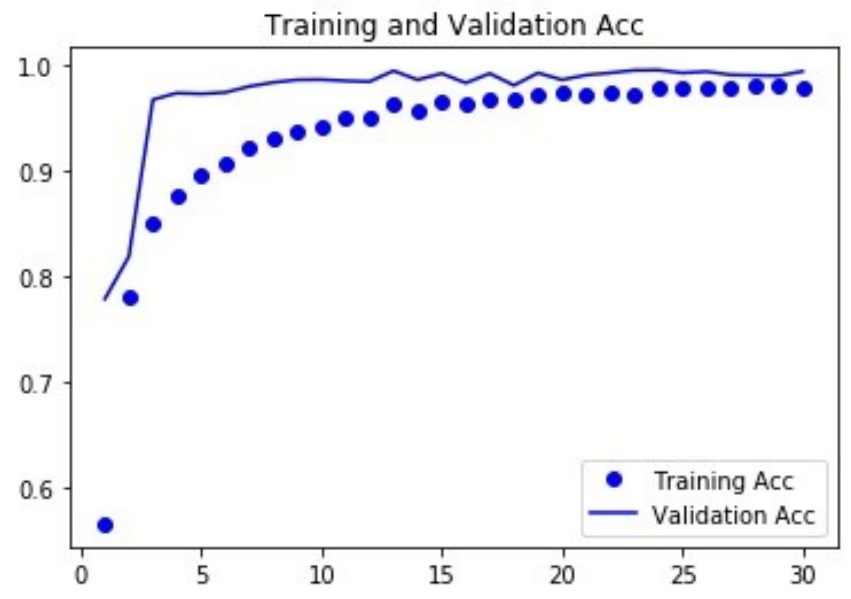

Fig. 2: Accuracy

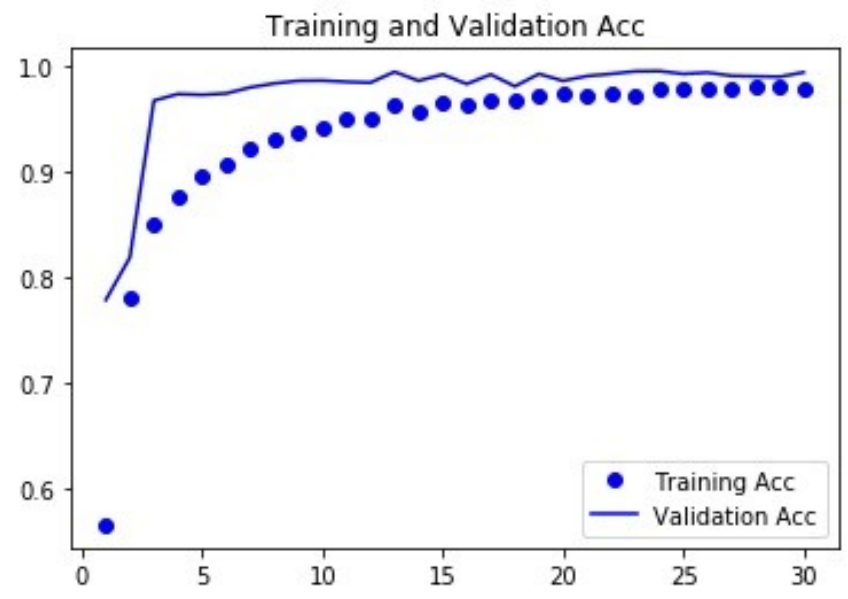

Fig. 3: Loss

them in the model. So, when videos are taken through the webcam and the collected frames are resized. In order to compare the frames with the trained frames, those frames are need to be in a particular size. The frames will be in sequence to predict the correct result. By comparing these frames, predicting the respective label with respect to the corresponding frames are tried. Fig. 4, show few of the gesture that the classifier has been able to predict by capturing the videos frames.

\section{RESUlts}

We have evaluated the performance of our dynamic hand gesture recognition system which we have trained by using the 27 different gestures that has been represented in more than 100 thousand ways. We have taken $35 \%$ of which for the validation purpose. Finally after obtaining the suitable match with respect to the hand gesture action, associates the gesture action with the assigned functionality that results in launching the application. Some screenshot of the end result with a video playing in the background are given in Fig. 5. There are still some issues are there in case some noises are there in the background. We are improving by focusing it on the hand movements only.

\section{CONClusion}

In the present day scenario the demand for system that can run on hand gesture recognition have grown rapidly. From the model it can be concluded that the system can detect hand gesture in real time. By showing meaningful hand gesture movements the system will detect the gestures and will perform its function according to the command that has been assigned. The system will be able to detect various gestures of multiple users. CNN as being the most reliable algorithm is used that provides high accuracy in real-time in detecting hand gestures. Although there are still some issues with the background noise during the frame collection that can eradicated in future.

\section{REFERENCES}

[1] P. C. Pankajakshan and B. Thilagavathi, "Sign language recognition system," in 2015 International Conference on Innovations in Information, Embedded and Communication Systems (ICIIECS). IEEE, 2015, pp. $1-4$.

[2] K. S. Varun, I. Puneeth, and T. P. Jacob, "Hand gesture recognition and implementation for disables using cnn's," in 2019 International Conference on Communication and Signal Processing (ICCSP). IEEE, 2019, pp. 0592-0595.

[3] D.-S. Tran, N.-H. Ho, H.-J. Yang, E.-T. Baek, S.-H. Kim, and G. Lee, "Real-time hand gesture spotting and recognition using rgb-d camera and 3d convolutional neural network," Applied Sciences, vol. 10, no. 2, p. 722,2020

[4] N. Alnaim, "Hand gesture recognition using deep learning neural networks," Ph.D. dissertation, Brunel University London, 2020.

[5] P. Xu, "A real-time hand gesture recognition and human-computer interaction system," arXiv preprint arXiv:1704.07296, 2017.

[6] M. Z. Islam, M. S. Hossain, R. ul Islam, and K. Andersson, "Static hand gesture recognition using convolutional neural network with data augmentation," in 2019 Joint 8th International Conference on Informatics, Electronics \& Vision (ICIEV) and 2019 3rd International Conference on Imaging, Vision \& Pattern Recognition (icIVPR). IEEE, 2019, pp. 324-329.

[7] P. M. Thwe et al., "ihand gesture detection and recognition system: A critical review," Ph.D. dissertation, MERAL Portal, 2019.

[8] X. Yingxin, L. Jinghua, W. Lichun, and K. Dehui, "A robust hand gesture recognition method via convolutional neural network," in 2016 6th international conference on digital home (ICDH). IEEE, 2016, pp. 64-67.

[9] G. Plouffe and A.-M. Cretu, "Static and dynamic hand gesture recognition in depth data using dynamic time warping," IEEE transactions on instrumentation and measurement, vol. 65, no. 2, pp. 305-316, 2015.

[10] P. Molchanov, S. Gupta, K. Kim, and J. Kautz, "Hand gesture recognition with $3 \mathrm{~d}$ convolutional neural networks," in Proceedings of the IEEE conference on computer vision and pattern recognition workshops, 2015, pp. $1-7$. 


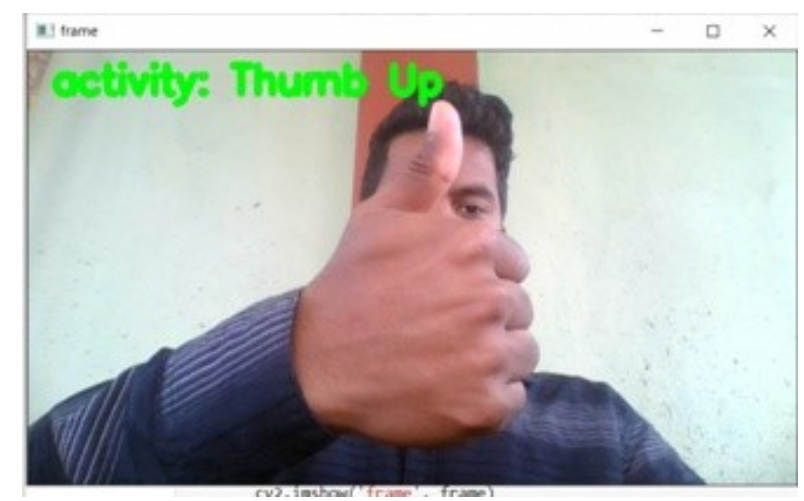

(a) Thumb up gesture

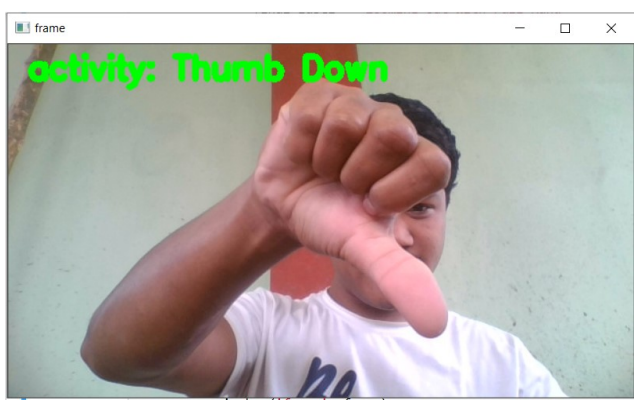

(c) Thumb down gesture

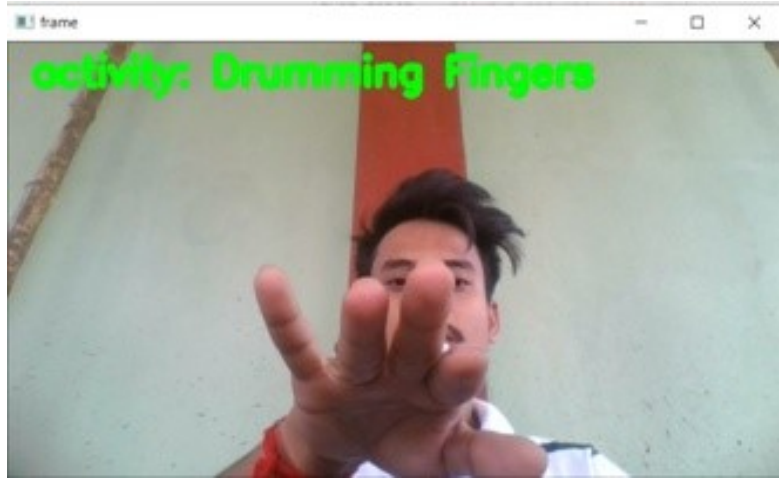

(b) Drumming gesture

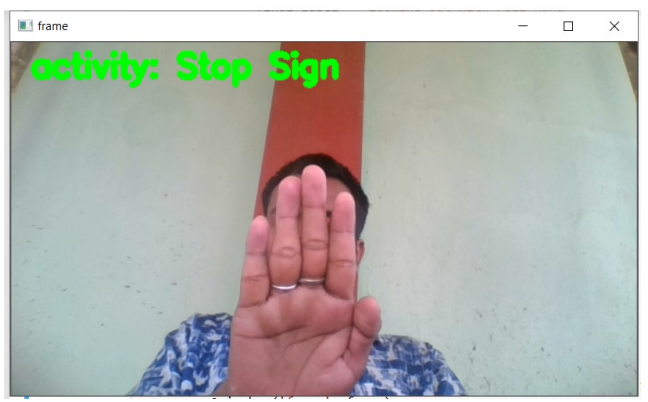

(d) Stop gesture

Fig. 4: Screenshots of system recognizing gestures. 


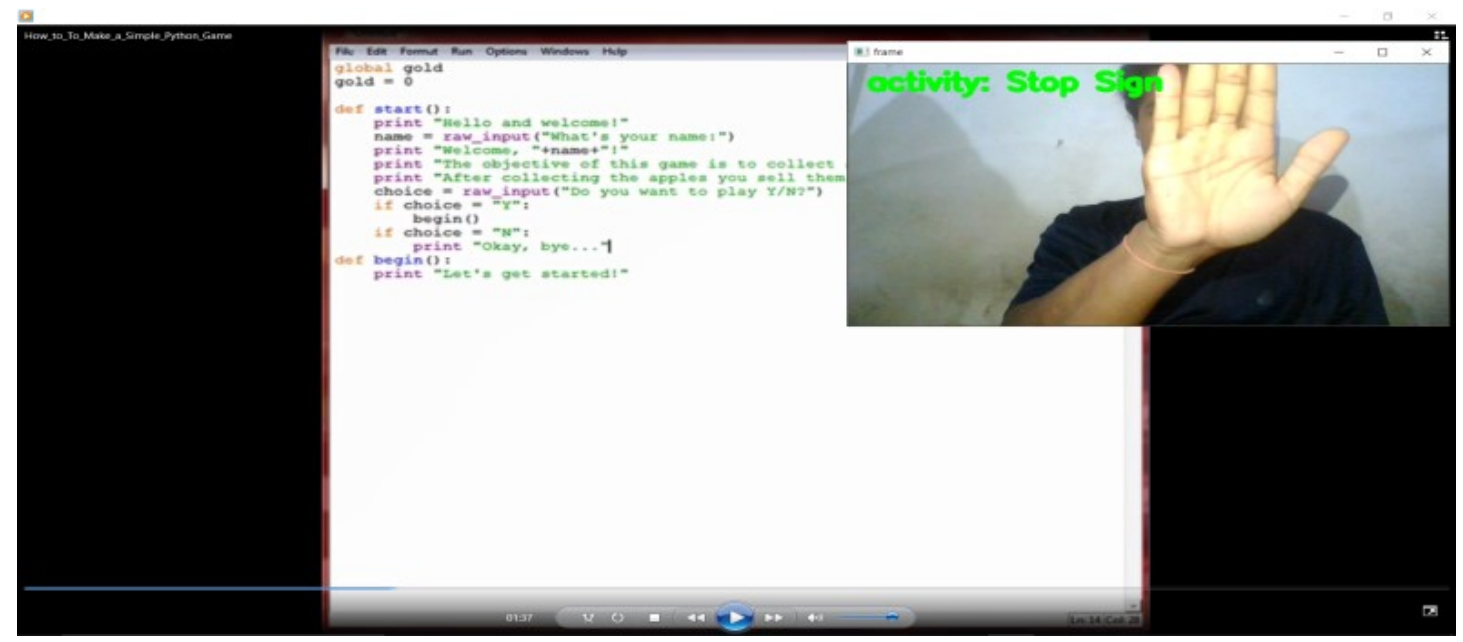

(a) Thumb up gesture

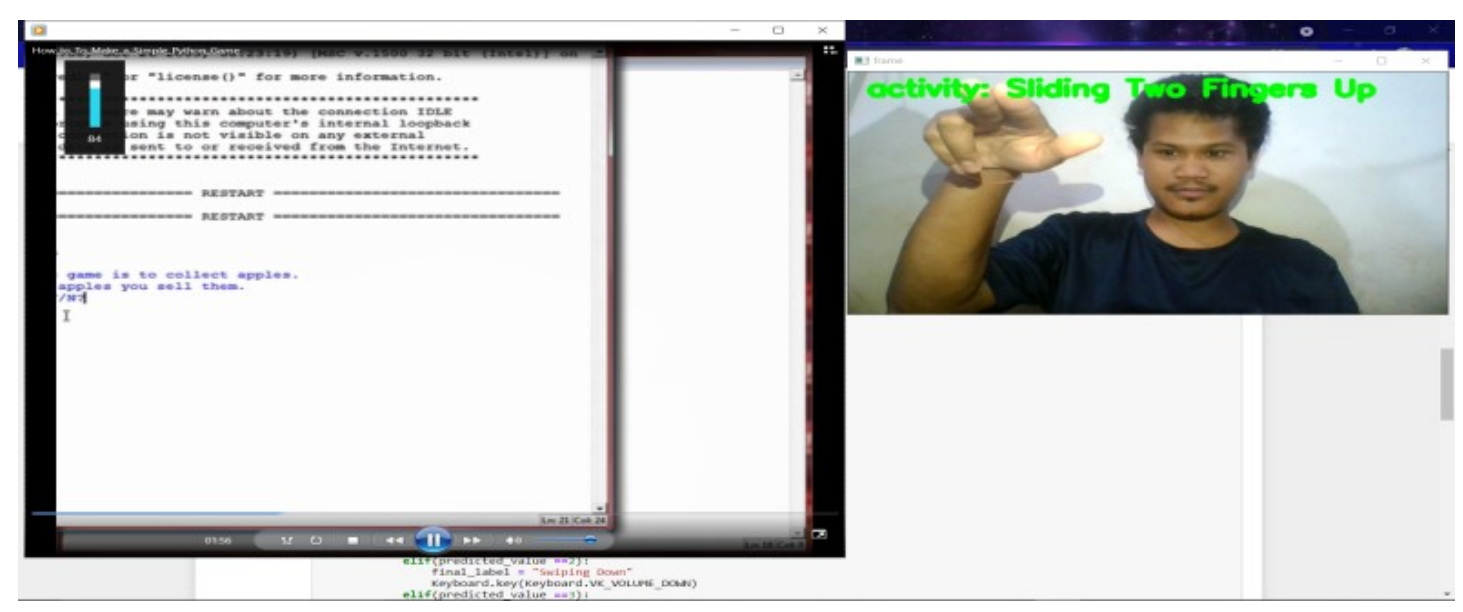

(b) Drumming gesture

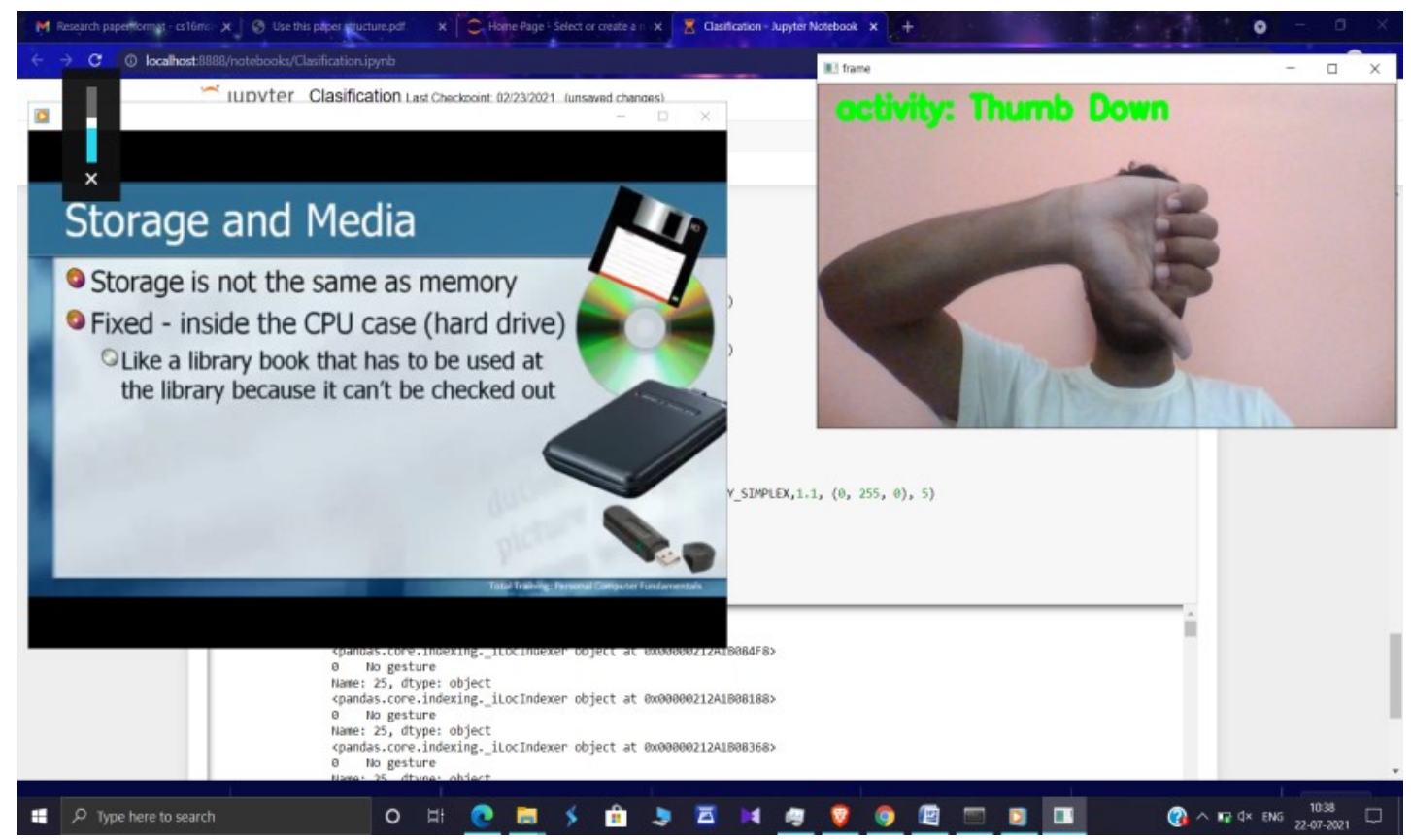

(c) Thumb down gesture

Fig. 5: Screenshots of working system. 